\title{
Epistemologia
}

\section{Da ciência do periódico à "ciência da liderança nacional-socialista": como os estudos de imprensa adotaram o regime nazista na Alemanha'}

From Newspaper Science to "NS-Führungswissenschaft": How the discipline of newspaper studies in Germany adopted to the Nazi Regime

Stefanie AVERBECK-LIETZ

Professora de Comunicação e Estudos de Mídia do Centro de Pesquisa de Mídia, Comunicação e Informação da Universidade de Bremen. Bremen, Alemanha. <averbeck.lietz@uni-bremen.de>

\section{RESUMO}

$\mathrm{O}$ artigo apresenta ao leitor de língua portuguesa alguns aspectos da história das ciências da comunicação na Alemanha, das origens até os anos 1960. Sublinhando as preocupações da atual geração em reavaliar seu passado, três momentos são analisados. Dominante no período que precedeu a ascensão do nazismo, o primeiro se caracteriza pelo desenvolvimento de uma abordagem empírica e interdisciplinar. O segundo revela o modo como o campo se submeteu ao novo regime e com ele colaborou, interrompendo sua atividade científica. O terceiro corresponde à situação do pós-Guerra e se caracteriza pelas tentativas de modernizar a área, adotando os conceitos e métodos de pesquisa originados dos Estados Unidos. A conclusão dá sinal de que o ciclo de reavaliação do passado está, agora, em vias de encerramento nas ciências da comunicação da Alemanha.

Palavras-chave: Ciências da Comunicação - Alemanha. História da Pesquisa em Comunicação. Mídia e Política - Alemanha.

\section{ABSTRACT}

Written to the Portuguese audiences, this article reveals some historical features of communication research in Germany from its origins to the 1960 's. It acknowledges the concerns of the contemporary generation toward a revaluation of his disciplinary past in order to analyze three phases. Predominating during the period before the take over of the state by the Nazis, the first one has characterized by the development of an empirical and interdisciplinary approach about the matter. The second one was marked by the subordination of the field by the regime and the collaboration that many of their representatives gave to it, paralyzing its scientific activities. The last one has arisen after the War and was characterized by the efforts to modernize the area, adopting concepts and methods coming from the United States. Some signs may lead us to think that the historical revaluation of the past is virtually concluded among the German researchers, suggests the article at the end. Keywords: Communication Studies - Germany. Communication Studies History. Media and Politics - Germany. 
$\mathrm{H}$ á mais de dez anos, Arnulf Kutsch e eu desenvolvemos um modelo acerca das etapas de desenvolvimento dos estudos de comunicação alemães ${ }^{2}$. Identificamos, então, seis fases:

1. Identificação dos problemas centrais de pesquisa e desenvolvimento de planos de estudo acadêmicos (1916-1925).

2. Definição e análise dos tópicos de pesquisa e formação de estudiosos (1925-1935).

3. Estabilização do processo de institucionalização, por meio de sua adaptação ao estado nazista (1933-1945).

4. Reconstrução da disciplina, após o colapso do nazismo (1945-1960).

5. Redefinição da disciplina como pesquisa social científica (1960-1980).

6. Instalação de novos métodos e teorias em meio a um mundo comunicativo em mudança (1990-atual).

No que segue, focarei nos estágios dois, três e quatro.

\section{A 1a fase: do início à República de Weimar}

A história da ciência do periódico (Zeitungwissenschat), na Alemanha, remonta ao final do século XIX e início do seguinte, quando os jornalistas, tanto quanto os sociólogos e economistas, começaram a pensar sobre a imprensa de massas no mundo moderno. Para fins de análise, observava-se nesse universo uma formação urbana, industrial e letrada, inserida em uma conexão transnacional que se estruturava com o amparo da ciência. Ninguém menos do que Max Weber (1864-1920) lançou argumentos nessa direção com seu famoso plano de pesquisa sobre a imprensa de $1910^{3}$. Weber tinha um novo objeto de pesquisa em mente: a imprensa de massas. Porém, foi só a partir de Ferdinand Tönnies (1855-1936), com seu famoso livro Crítica da opinião pública (Die Kritik der Öffentlichen Meinung, 1922), que, de fato, se começou a inspirar a geração mais jovem de sociólogos e estudiosos do jornalismo. Tönnies observou o 
surgimento dos movimentos de opinião como forma de "consciência social" (1922, p. 573) da luta pelo direito civil das mulheres e da humanidade, do direito à liberdade de expressão e da liberdade de imprensa, tanto nos Estados Unidos quanto nos países europeus. Pouco mais de uma década depois, ele foi, por razões políticas, afastado de sua cátedra na Universidade de Kiel pelos novos administradores nazistas. Durante toda a República de Weimar, não fora, porém, apenas ele que influenciara seus jovens discípulos a encarar a diferença entre dois conceitos de opinião pública. Isto é, a "Opinião pública”, com letra maiúscula, unificada, consensual e elitista da chamada "Repúblicas das Letras" (Gelehrtenrepublick); e as opiniões públicas de tipo plural, divergentes, que peleavam para obter hegemonia sobre a opinião pública (Tönnies, 1922). Tanto os jovens sociólogos que se educaram com ele quanto o círculo que o citava apegaram-se a essas ideias e perguntaram se a moderna imprensa de massas é um fator influente no processo de construção da opinião.

Ainda em 1928, com Gerhard Münzer, em 1930, com Walter Auerbach, e, em 1933, com Ernest Manheim, esboçou-se uma visão do processo comunicacional como bi ou multidirecional, indo-se além do esquema estímulo-resposta então predominante. Tomando os termos do livro de Tönnies, esses autores e outros esboçaram cenários dinâmicos para pensar o assunto: leitores que construíam opiniões gasosas ou fluídas, de acordo com sua socialização primária de longa duração, em vez de se apoiarem na imprensa cotidiana (Münzer, 1928); leitores que estavam inseridos em padrões de comportamento e atitude grupal (Auerbach, 1930), tanto quanto em seus mecanismos de controle (Willems, 1930); jornais que se orientavam pela opinião de setores do público (Carlé, 1931); leitores que eram em si mesmos comunicadores, como foi em especial o caso da esfera pública burguesa no século XVIII (Manheim, 1933). Ernest Manheim (1900-2002) foi 
obviamente o mais brilhantes desses jovens pensadores ${ }^{4}$. Manheim concebia todos os processos comunicacionais como uma rede tramada por um comunicador (portador de intenções), um destinatário (com expectativas) e um conteúdo (adaptado a essas intenções e expectativas), em meio a um dispositivo espaço-temporal de comunicação que se iniciava dentro de um grupo e daí se irradiava para outros, da comunicação interpessoal para a imprensa de massa (Manheim, 1933; Averbeck, 1999).

Este "processo de pensamento orientado" (Averbeck, 1999) da geração de estudantes de doutorado e professores-assistentes de 1930, inspirado por sociólogos como Max Weber, Ferdinand Tönnies e também Karl Mannheim (1895-1947), que ofereceu curso sobre a ciência do jornal na Universidade de Heidelberg, no início dos anos 1930 (Averbeck, 1999, p. 226), era novo e desafiador. Por que, porém, os estudiosos da imprensa se voltaram pesadamente para a sociologia? Houve razões analíticas, mas também devido a um problema estrutural: não havia autorização para conferir o título de doutor aos que seguiam os estudos de jornalismo na Universidade.

A Universidade de Leipzig era uma exceção, tendo obtido esse direito em 1926. Por essa razão, professores de jornalística como Emil Dovifat (1890-1968), em Berlim, ou Karl d'Ester (1881-1960), em Munique, supervisionavam dissertações doutorais que tinham de ser avaliadas por professores de sociologia (como Alfred Vierkandt o havia feito para validar o PhD de Emil Willems, em Berlim) ou economia (como Salomon Paul Altmann e Emil Lederer para o caso de Heidelberg) (Averbeck, 1999). Vista dos dias de hoje, esta falta de autoridade para conferir diplomas era uma desvantagem que, todavia, incentivou a interdisciplinaridade. Por volta do início dos anos 1930, podemos afirmar que havia uma crescente interdisciplinaridade entre os meios acadêmicos da ciência do periódico e a sociologia. 


\section{A 2a Fase: da República de Weimar ao Estado nazista}

A promissora decolagem em direção a um moderno entendimento dos processos de comunicação verificada nos estudos de jornalismo foi suspensa a partir de 1933 (Averbeck, 1999; 2001; 2008). Para tanto, várias razões convergiram:

- A ciência do periódico se institucionalizou, às vezes de maneira forçada, com o financiamento que recebeu do Ministro da Propaganda, ao mesmo tempo em que perdia seus conceitos teóricos e metodológicos (Averbeck, 1999; Averbeck e Kutsch, 2002; Kutsch, 2002).

- Suas ideias foram absorvidas ideologicamente: conceitos inspiradores foram suprimidos. No caso, "opinião pública”, por exemplo, foi reduzido a Volksmegeinschaft (comunidade popular), um conceito típico do pensamento fascista (Kurth e Hollmann, 1940). A Associação dos Pesquisadores de Jornalismo (Deutscher Zeitungwissenschaflicher Verband - DZV) passou a condenar a abordagem sociológica, estabelecendo que as dissertações com foco sociológico não seriam mais bem recebidas e, em alguns casos, tornar-se-iam declaradas "opiniões privadas" de seus autores, permanecendo sem permissão para serem publicadas, como ocorreu com Heinrich Arimond, em Munique (Averbeck, 1999, p. 134).

- A propaganda foi legitimada como forma de comunicação (Six, 1936; Kurth e Hollmann, 1942).

- O antissemitismo, a denúncia e o ódio racial, impuseram-se com a denúncia por parte dos professores dos estudantes que cometiam "crimes raciais" (Averbeck, 1999, p. 111). Professores como Hans Amandus Münster chegavam a forçar seus estudantes a participar de "pesquisas" racistas a respeito da imprensa judaica (Ehrich, 1992, p. 92; Jedraszczyk, 2011, p. 199). 
- Houve professores que simpatizaram abertamente com ou apoiaram ativamente o III Reich. Jovens, fizeram grandes carreiras, embora suas teses de doutoramento tenham sido muito fracas. Karl Kurth (1910-1981), posteriormente Diretor do Instituto de Jornalística da Universidade de Viena, fundado durante a época do Anchluss, foi um deles (Kutsch, 1984; Kniefacz, 2008).

- Da parte da geração mais velha dos especialistas em ciência do periódico, a primeira strictu sensu, houve uma espécie de adaptação oportunista às novas circunstâncias, como se pode ver nos casos de Emil Dovifat (1890-1968) e Karl d'Ester (1861-1960) (Meyen e Löblich, 2006; Bohrmann, 2004; Pöttker, 2004).

- Os professores assistentes e os estudantes de doutorado orientados no sentido da pesquisa interdisciplinar fugiram ou emigraram. Gerhard Münzner foi para a Palestina; Emil Willems passou mais de dez anos no Brasil, antes de ir para os Estados Unidos; Walter Auerbach foi para a Grã-Bretanha; Ernest Manheim saiu para aquele país via a Hungria, antes de ir para os Estados Unidos (Kutsch, 1988; Averbeck, 1999, 2001).

Gerhard Münzner (1904-1958), mais tarde chamado Gershon Meron, afastou-se da área, passando a trabalhar para a Federação Trabalhista Judaica na Palestina, antes de se tornar diretor de uma companhia de energia elétrica em Israel (Averbeck, 1999). Walter Auerbach (1905-1975) integrou a resistência ao nazismo, antes de fazer carreira no Ministério do Trabalho alemão, no final dos anos 1960 (Averbeck, 1999). Emil Willems (1905-1997) acabou lecionando na Universidade Vanderbilt, no Tennessee. Ernest Manheim (1900-2002), sobrinho mais jovem de Karl Mannheim, juntouse a London School of Economics, em 1935, e, dois anos depois, ao Departamento de 
Sociologia da Universidade de Chicago, antes de se radicar no mesmo departamento da Universidade do Missouri, em Kansas City, em 1938.

A ciência do periódico nacional-socialista, como outros ramos disciplinares da universidade alemã, comprometeu-se ativa, intensa e inequivocamente com o "III Reich" (Kutsch, 1987, 2010). E isso vale para a pesquisa tanto quanto para a formação e treinamento de jornalistas (Averbeck, 1999, p. 142). A Zeitungswissenchaft se tornara espaço privilegiado para jornalistas e políticos nazistas de alto escalão fazerem carreirismo, como ocorreu com Franz Alfred Six (1909-1975), oficial do serviço secreto das SS (Sicherheitsdienst der SS), professor de ciência jornalística em Königsberg, a partir de 1937, Kaliningrado, atualmente (Hachmeister, 2004).

Quem fez a ligação entre a ciência do periódico e a administração nazista para chegar até o Ministério da Propaganda, foi Walther Heide (1884-?). Heide fora secretário de imprensa do Gabinete de Relações Exteriores durante da República de Weimar e, em seguida, porta-voz do Governo Papek-Hugenberg-Hitler, antes de se tornar chefe do Escritório de Imprensa Internacional, seção de propaganda para o estrangeiro do nacional-socialismo, em 1933. Em 1934, Heide recebeu o título de professsor-honorário da Universidade Técnica de Berlim e se tornou líder da Deutscher Zeitungswissenschaftlicher Verband, União dos pesquisadores de jornalismo alemães (Bohrmann e Kusch, 1981; Kutsch, 2010). Heide organizou a ciência do jornalismo de acordo com o princípio da liderança (Führerprinzip), chegando a construir uma organização estudantil conformista para apoiá-la. Ele obteve fundos para expandir a infraestrutura dos institutos da área diretamente do Ministério da Propaganda. Ocorreu assim especialmente em Munique, onde seu grande amigo, Karl d'Ester, recomendado por ele, fora instalado como professor, em 1934 (Kutsch, 2010). O Instituto de Munique se tornou conhecido em seguida como Doktorfabrik - fábrica de doutores (Meyen, 2004; Meyen e Löblich, 2006). 
Heide não era um fanático pelo estado nacional socialista, mas um funcionário eficiente e superambicioso, que construiu um sistema de distribuição de cargos, bolsas e honrarias, a fim de comandar a comunidade dos pesquisadores em jornalismo. Junto com o líder da imprensa política do regime, Otto Dietrich (1887-1953), e com a ajuda financeira do Ministério da Propaganda, ele conseguiu criar institutos para a área em Viena, Praga e Königsberg, durante o período em que essas cidades estiveram sob ocupação alemã (Kniefacz, 2008). Que fim Heide teve não está claro. Alguns dizem que ele morreu em uma prisão russa em 1957, mas isso é incerto.

\section{A espiral do silêncio na ciência}

É vergonhoso, mas foi tarde que os estudos de comunicação alemães olharam para seu passado. Foram pesquisadores da segunda geração, a geração de netos, por assim dizer, dos professores da época do III Reich, em especial Hans Bohrmann (nascido em 1940) e Arnulf Kutsch (nascido em 1949), que recuperaram grande parte dessa história esquecida (Bohrmann; Kutsch, 1981; Kutsch, 1985, 1987, 2011; Borhmann, 2004). Na Áustria, este trabalho de desmascaramento da história da ciência do periódico tem sido feito por Wolfgang Duchkowitsch (nascido em 1942) e Fritz Hausjen (nascido em 1959).

Depois de 1945, começara uma ruptura e uma rejeição do passado, isto é, uma depuração por desconhecimento (Langenbucher, 2004; Kutsch, 2006; Koenen, 2008). “Desnazificados" pela administração aliada, os velhos d'Ester, em Munique, e Dovifat, em Berlin, retomaram suas atividades. Dovifat (re)fundou o Instituto de Jornalística na nova Universidade Livre. Com base em minha própria pesquisa nos arquivos, posso dizer: mesmo que tenha sido uma figura problemática por ter seguido com sua cátedra durante a era nazista, ele, por certo, não era um antissemita. Dovifat chegou a mentir para proteger seu assistente acadêmico Hans Traub (1901-1943), que tinha bisavôs judeus (Averbeck, 1999). 
Depois da II Guerra Mundial, a maior parte dos institutos, todavia, fechara completamente, devido à ideologia nazista de seus ex-diretores. Dos 17 existentes antes dela, apenas três (Munique, Münster e Berlin, este instalado na nova Universidade Livre) sobreviveram na Alemanha Ocidental (Stöber, 2004b, p. 123). Leipzig, o outro que restou, teve história diferente. Os nazistas despediram o segundo diretor, Erich Everth (1878-1934), que havia sucedido o famoso economista Karl Bucher (1847-1930), que o fundara, em 1918. No lugar dele, os nazistas colocaram o ideologicamente conformista Hans Amandus Münster (1901-1965). Após a Guerra, o Instituto tornou-se o centro de formação dos jornalistas na Alemanha Oriental. Hermann Budzislawski (1901-1978), conhecido por ser um dos editores do jornal Die Weltbühme e que emigrara para os Estados Unidos durante a era nazista, foi seu primeiro diretor ${ }^{5}$.

\section{A 39 Fase: renovação com esquecimento}

Depois de 1945, na Alemanha Ocidental, a ciência do periódico aparentemente se encontrava, portanto, numa rua sem saída, devido a seu passado, em parte criminoso, em parte altamente ideológico. Ocorrera um vínculo direto entre a formação acadêmica e a inserção dos formandos na administração do estado nazista, especialmente no Instituto de Leipzig, dirigido por Münster, e no de Königsberg, dirigido por Franz Alfred Six. Ambos planejaram e ajudaram seus estudantes a fazer carreira política, inclusive no Serviço Secreto das SS (Hachmeister, 2004). Apesar disso, Six e Münster conquistaram novos terrenos após a Guerra, entrando no emergente setor de pesquisa de mercado e publicidade. Six, por exemplo, trabalhou para a Porsche (Wagner, 2013; Hachmeister, 2002, 2004; Urban e Herpolsheimer, 1984). Ele tinha sido punido com 20 anos de prisão durante o Julgamento de Nuremberg por crimes de guerra, mas acabou anistiado em 1952 pelo Alto Comissariado Aliado (Hachmeister, 2002, 2004). 
Durante os anos 1960, houve uma certa renovação, que tirou a área da estagnação que representara a continuidade não muito produtiva das cátedras de Dovifat, em Berlin, e d'Ester, em Munique (Stöber, 2004; Koenen, 2008). As razões para tanto foram as seguintes:

- As Forças Aliadas promoveram uma desnazificação, banindo sua ideologia (mas sem muita reflexão crítica a respeito do passado nazista da comunidade acadêmica) (Hardt, 2004).

- (Re)importaram-se as teorias e metodologias de médio alcance, do tipo "líder de opinião", ao mesmo tempo em que se adotou a epistemologia positivista e quantitativa dos estudos de comunicação norte-americanos (Reimann, 1990; Löblich, 2010).

- A Publizistikwissenschaft se institucionalizou, ampliando o foco dos estudos jornalísticos, para incluir a radiodifusão e demais tipos de comunicação pública, via a contratação de novos professores, oriundos principalmente da prática profissional e de outras disciplinas, inclusive estrangeiros (Meyen e Löblich, 2006; Löblich, 2010).

Na Universidade de Münster, Walter Hagemann (1900-1964), um ex-jornalista católico, doutorado em História, pela Universidade de Berlin, começou a lecionar Publizistikwissenchaft, em 1946. Ele foi um dos primeiros acadêmicos alemães a estudar o sistema de propaganda nazista (Hagemann, 1948). Membro da União Cristã Alemã, o partido de Konrad Adenauer, ele, porém, trabalhou contra a política de integração ao Ocidente deste último, tornando-se um dos líderes do movimento antinuclear na Alemanha Ocidental. Em 1959, perdeu sua cátedra por razões políticas, mas também por ser acusado de relacionamento com suas estudantes. Partindo para a Alemanha Oriental, lá faleceu poucos anos depois (Wiedemann, 2012). 
Dentre as novas lideranças da Publizistikwissenschaft nessa época estava o sociólogo e editor holandês Henk Prakke (1900-1992), que fugira dos nazistas para a cidade de Amsterdam no início dos anos 1940, para ser preso, pela Gestapo, em 1945. Em 1960, foi nomeado professor de ciência publicística para a Universidade de Münster, passando a desenvolver um rico modelo teórico, baseado em um entendimento antropológico da comunicação muito mais amplo do que o de Emil Dovifat, Karl d'Ester e, mesmo, de seu predecessor em Münster, Walter Hagemann. Durante a rebelião estudantil, novamente teve de fugir da Alemanha. Alguns estudantes cercaram seu escritório, com o objetivo de reduzir a autoridade do professorado. Prakke viu nisso uma agressão, um insulto, considerando que haviam se passado apenas 15 anos desde o final da II Guerra Mundial: por isso, ele deixou o Instituto (Kutsch, Hemels e Schmolke, 2000; Klein, 2006; Meyen e Löblich, 2006).

Elisabeth Noelle-Neumann (1916-2010) começou a construir um novo instituto na Universidade de Mongúcia, em 1964, após anos de experiência com pesquisa de opinião e pesquisa de mercado no Instituto Allensbach, que fundara em 1947. Entre 1933 e 1945, ela fora estudante de Emil Dovifat, em Berlin e chegou a ser bolsista do Serviço Alemão de Intercâmbio Universitário na Universidade do Missouri, Estados Unidos. Também trabalhou como jornalista no Das Reich, no Deutsches Allgemeine Zeitung, no Berliner Tageblatt e no Frankfurter Zeitung. Acusada de oportunismo e antissemitismo por isso, foi depois fortemente criticada no pós-Guerra (Wendelin, 2013; Becker, 2013).

Baseando-se em sua vasta pesquisa eleitoral, ela desenvolveu o famoso conceito de espiral do silêncio, para dar conta da pressão do público sobre os processos de formação da opinião individual fortemente influenciada pela televisão (Schenk, 2007). Por um lado, seu modelo foi frutífero para a pesquisa empírica pelo mundo afora, mas de outro muito criticado: sua tipificação do indivíduo como ser fraco e passivo diante 
da pressão da opinião pública foi comparada a dos chamados seguidores (Mitläufer), conforme os caracterizam Adorno e Horkheimer no livro A Personalidade autoritária. Em seu modelo de opinião pública, a deliberação e a racionalidade do discurso não estão em questão.

O choque entre Noelle-Neumann e o erudito norte-americano Christopher Simpson, a respeito do passado da primeira, gerou grande interesse na mídia dos dois países, chegando às páginas do Der Spiegel e do The New York Times. Pouco conhecido, talvez por não se encaixar bem na visão que dela tinha o público mais crítico, é o fato que durante os 1950, ter trabalhado de maneira muito próxima com Theodor Adorno em um projeto de pesquisa de mercado e em seu projeto de habilitação para lecionar na Universidade (Grube, 2013). Durante toda sua vida, negou as acusações de ter sido nazista ou possuir convicções fascistas (Meyen e Löblich, 2006; Wendelin, 2013). Noelle segue sendo uma figura ambígua, havendo necessidade de mais pesquisa sobre sua biografia científica e jornalística, tanto quanto seu papel no campo da pesquisa de mercado e como consultora política de gente como o chanceler Helmut Kohl (Meyen e Löblich, 2006). Qualquer que seja o segmento da ideologia nazista que tenha ou não influenciado sua vida e obra, o fato é que a autora foi parte da elite que manteve a continuidade entre o início dos anos 1940 e a jovem República Federal Alemã (Becker, 2013; Fuchs, 2012; Grube, 2012).

Outras duas personalidades conhecidas pertenceram ao campo, embora não estivessem institucionalmente dentro dele: Gerhard Malettzke (1922-2010) e Otto Groth (1875-1965). Groth é conhecido no Brasil pela sua definição da imprensa como órgão da atualidade, universalidade, periodicidade e publicidade, formulada em 1928. Judeu, foi banido da Universidade de Munique, em 1934, e impedido de lecionar. Mais tarde, seu extenso trabalho A imprensa como poder cultural (Die unerkannte Kultumacht) tornou-se muito influente por causa do emprego do conceito 
de mediação (vermittelte Mitteilung) (Langenbucher, 1998; Brosda, 2008; Hepp, 2012), parcialmente traduzido como "periodismo" em língua portuguesa (Groth, 2011).

Maletzke formulou, por sua vez, um modelo de comunicação que há décadas vem sendo recapitulado pelos estudiosos alemães: o chamado esquema do campo da comunicação de massas (Feldschema der Massenkommunication), que integra o comunicador e a pesquisa orientada para o usuário em um mesmo modelo. Como Prakke e Noelle-Neumann, ele traduziu a pesquisa em comunicação norte-americana para a comunidade acadêmica alemã. Ainda em 1962, começou a trabalhar no campo da comunicação intercultural, entrando em tópico negligenciado por seus colegas, devido à fixação, então em voga, por assuntos nacionais (Lietz e Klein, 2009). Maletzke nunca teve cátedra na Alemanha. A partir de 1972, ajudou a construir o Centro Asiático de Comunicação de Massa e Informação, em Singapura (Meyen e Löblich, 2006).

\section{Parecer}

Quando se escreve a história dos estudos de comunicação, encontramos vários enfoques: institucional, biográfico, contextual e geracional ${ }^{6}$. No entanto, existe um claro déficit: sempre que se pesquisa carreiras acadêmicas (inclusive as do período entre guerras), o foco se fixa nas carreiras bem sucedidas, das quais o melhor exemplo é a do emigrante Paul Felix Lazarsfeld (1901-1976) (Langenbucher, 1988). A pesquisa feita nas margens dos estudos de comunicação, não menos daquelas concernentes às pesquisadoras do sexo feminino, é muito difícil de fazer (Rowland; Simonson, 2013) e, por isso, ainda é muito fraca. A história da corrente principal também é sempre uma história de supressões. Que os estudos de comunicação alemães tenham agora quase 40 anos de tradição no campo da história de sua disciplina se deve principalmente ao interesse despertado pelo cruel passado dos estudos periodísticos na era nazista ${ }^{7}$. Atualmente, porém, as janelas a respeito estão fechando e a comunidade de investigadores alemães 
está tentando se tornar mais inter e transnacional em perspectiva (Schäfer, 2012; Averbeck-Lietz, 2011 e 2012; Löblich e Scheu, 2011), inserindo-se em uma tendência internacional crescente e promissora (Blumler, McLeaod e Rosengren, 1992; Mattelart e Mattelart, 2002; Katz, Peters, Liebes e Orloff, 2003; Scannell, 2007; Thussu, 2009).

O conhecimento a respeito do avanço dos estudos comunicacionais em todo o mundo é relevante não apenas por razões históricas, mas também para se entender como diferentes culturas de pesquisa fazem frente a tendências globais semelhantes que vão além da mídia tradicional em uma era de digitalização e, assim, saber que recursos teóricos e metodológicos suas comunidades trazem de seus respectivos bastidores históricos (Averbeck-Lietz, 2014).

\section{REFERÊNCIAS}

AUERBACH, Walter. Presse und Gruppenbewußtsein. Berlin: Laubsche Verlagsbuchhandlung, 1930.

AVERBECK, Stefanie. Kommunikation als Prozess. Soziologische Perspektiven in der Zeitungswissenschaft, p. 1927-1934. Münster: LIT, 1999. . The Post-1933 Emigration of Communication Researchers from Germany. The Lost Works of the Weimar Generation. In: European Journal of Communication, v. 16, n. 4, p. 451-475, 2001.

Comparative History of Communication Studies: France and Germany. In: The Open Journal of Communication, v. 2, n. 1, p. 1-3, 2008.

AVERBECK, Stefanie; KUTSCH, Arnulf: Thesen zur Geschichte der Zeitungs- und Publizistikwissenschaft 1900-1960. In: Medien E Zeit, 17. Jg. (2002), Nr. 2-3, S. 57-67.

AVERBECK-LIETZ, Stefanie. French and Latin American Perspectives on Mediation and Mediatization: A lecture note from Germany. In: Empedocles: European Journal for the Philosophy of Communication, v. 3, n. 2, p. 177-195, 2011.

. Communication Studies beyond the National. Connections and Disconnections between Research Communities and How to Study Them. In: Global Media Journal, German Edition, v. 2, n. 2, Autumn/Winter, p. 1-10, 2012.

AVERBECK-LIETZ, Stefanie; KLEIN, Petra. Entwicklungs- und interkulturelle Kommunikation in der Funktionalen Publizistikwissenschaft. Henk Prakke und Franz-Josef Eilers. In: AVERBECK-LIETZ, Stefanie; 
KLEIN, Petra; MEYEN, Michael (Ed.). Historische und Systematische Kommunikationswissenschaft. Festschrift für Arnulf Kutsch. Bremen: Edition Lumière, S. 215-238, 2009.

BECKER, Jörg. Elisabeth Noelle-Neumann. Demoskopin zwischen NS-Ideologie und Konservatismus. Stuttgart: Schöningh, 2013.

BENEDICT, Klaus-Ulrich. Emil Dovifat. Ein katholischer Hochschullehrer und Publizist. Mainz: Grünwald, 1986.

BLUMLER, Jay; MCLEOD, Jack; ROSENGREN, Karl Erik. Comparatively Speaking: Communication and Culture across Space and Time. Newbury Park: London, 1992.

BOHRMANN, Hans; KUTSCH, Arnulf. Der Fall Walter Heide. Zur Vorgeschichte der Publizistikwissenschaft. In: Publizistik, v. 19/20, n. 3, p. 805-808, 1981. 1981.

. Karl d'Ester. Anmerkungen aus Anlaß seines 100. Geburtstages. In: Publizistik, v. 26, n. 4, p. 575-603,

BOHRMANN, Hans. Als der Krieg zu Ende war. Von der Zeitungswissenschaft zur Publizistik. In: DUCHKOWITSCH, Wolfgang/HAUSJELL, Fritz/SEMRAD, Bernd (Ed.). Zum Umgang mit der nationalsozialistischen Zeitungswissenschaft. Münster: LIT, 2004. p. 97-122.

BROSDA, Carsten. Diskursiver Journalismus. Journalistisches Handeln zwischen kommunikativer Vernunft und mediensystemischem Zwang. Wiesbaden: VS, 2008.

CARLÉ, Wilhelm. Weltanschauung und Presse. Eine Untersuchung an 10 Tageszeitungen. Leipzig: Hirschfeld, 1931.

DUCHKOWITSCH, Wolfgang; HAUSJELL, Fritz; SEMRAD, Bernd. Die Spirale des Schweigens. Zum Umgang mit der nationalsozialistischen Zeitungswissenschaft. Münster: LIT, 2004.

EHRICH, Ute. Die Judenfrage in der Presse. Forschung und Denunziation am Institut für Zeitungswissenschaft an der Universität Leipzig. In: RÖHR, Werner (Ed.). Faschismus und Rassismus. Kontroversen um Ideologie und Opfer. Berlin: Akademie-Verlag, 1992. p. 86-95.

ESCOSTEGUY, Ana Carolina; RÜDIGER, Francisco. Brazilian Research in Communication: Historical Synopsis and Reflexive Trends in Academic Work in an Emerging Country. In: AVERBECK-LIETZ, Stefanie (org.): Kommunikationswissenschaft im internationalen Vergleich: Communication Studies under International Comparison. Wiesbaden: VS, 2014.FUCHS, Christian; BECKER, Jörg. Elisabeth Noelle Neumann. Demoskopin zwischen NS-Ideologe und Konservatismus. In: TripleC, v. 11, n. 2, p. 310-317, 2012.

GROTH, Otto. O Poder Cultural Desconhecido. Fundamentos da Ciência dos Jornais. Petrópolis: Vozes, 2011.

GRUBE, Norbert. (2013) [Review of] Jörg Becker: Elisabeth Noelle-Neumann. In: H-Soz-Kult. Disponível em: $<$ http://hsozkult.geschichte.hu-berlin.de/rezensionen/2013-3-064>. Acesso em: 7 fev. 2014. 
HACHMEISTER, Lutz. Der Gegnerforscher. Die Karriere des SS-Führers Franz Alfred Six. München: C.H. Beck, 1998.

. Die Rolle des SD-Personals in der Nachkriegszeit. Zur nationalsozialistischen Durchdringung der Bundesrepublik. In: Mittelweg 36, v. 11, n. 2, p. 17-36, 2002.

. Presseforschung und Vernichtungskrieg. Zum Verhältnis von SS, Propaganda-Apparat und Publizistik. Münster: LIT, 2004. p. 67-80.

HAGEMANN, Walter. Publizistik im Dritten Reich. Ein Beitrag zur Methodik der Massenführung. Hamburg: Heitmann, 1948.

HARDT, Hanno. Social Theories of the Press. Constituents of Communication Research 1840s to 1920s. Oxford: Rowman \& Littlefield, 2001.

Am Vergessen Scheitern. Essay zur historischen Identität der Publizistikwissenschaft 1945-68. In: DUCHKOWITSCH, Wolfgang; HAUSJELL, Fritz; SEMRAD, Bernd (Ed.). Die Spirale des Schweigens. Zum Umgang mit der nationalsozialistischen Zeitungswissenschaft. Münster: LIT, 2004. p. 153-160.

HEPP, Andreas. Cultures of Mediatization. Cambridge: Polity, 2012.

JEDRASZCZYK, Jochen. Hans Amandus Münster und die Ideologisierung des Leipziger Instituts für Zeitungswissenschaft im Dritten Reich. In: Jahrbuch für Universitätsgeschichte, v. 14, p. 189-204, 2011.

KAPP, Wilhelm. Ernst Manheim: Die Träger der öffentlichen Meinung. In: Zeitungswissenschaft, v. 9, n. 4, p. 189-192, 1934.

KATZ, Elihu; PETERS DURHAM, John; LIEBES, Tamar; ORLOFF, Avril. Canonic Texts in Media Research. Are there any? Should there be? How about these? Cambridge: Polity, 2003.

KLEIN, Petra. Henk Prakke und die funktionale Publizistik: Über die Entgrenzung der Publizistik- zur Kommunikationswissenschaft. Berlin, Münster: LIT, 2006.

KNIEFACZ, Katharina. (2008). Zeitungswissenschaft in Wien 1900-1945. Die Institutionalisierung im Kontext der deutschsprachigen Fachentwicklung. Universität Wien [geschichtswiss. Diplomarbeit]. Disponível em: $<$ http://othes.univie.ac.at/1046/1/2008-08-08_0000987.pdf>. Acesso em: 12 fev. 2014.

KOENEN, Erik. Auf Schleichwegen in die neue Zeit? Anmerkungen zur Re-Konstituierung der Zeitungswissenschaft als Publizistik(wissenschaft) nach 1945. In: REHBERG, Karl-Siegbert (Ed.). Die Natur der Gesellschaft. Verhandlungen des 33. Kongresses der Deutschen Gesellschaft für Soziologie in Kassel 2006, p. 3310-3324, 2008.

. Die Begründung der Zeitungskunde als akademische Spezialität. Entwicklung ihrer Ideen-, Interessen und Institutionengestalt in Leipzig. In: AVERBECK-LIETZ, Stefanie; KLEIN, Petra; MEYEN, Michael (Ed.). Historische und systematische Kommunikationswissenschaft. Festschrift für Arnulf Kutsch. Bremen: Edition Lumière, 2009. p. 157-180. 
KUTSCH, Arnulf. Rundfunkwissenschaft im Dritten Reich. Geschichte des Instituts für Rundfunkwissenschaft an der Universität Freiburg. München, New York: K.G. Saur, 1985.

. Zeitungswissenschaftler im Dritten Reich. Sieben biografische Studien. Köln: Hayit, 1984.

. Karl Oswin Kurth (1910-1981). In: KUTSCH, Arnulf. Zeitungswissenschaftler im Dritten Reich. Sieben biographische Studien. Köln: Hayit, S. 215-243, 1984.

. Zeitungswissenschaftler im Dritten Reich. Sieben biographische Studien. Köln: Ertay Hayit, 1987.

. Die Emigration der Zeitungswissenschaft ab 1933. Anmerkungen zu einem vergessenen Thema. In: Medien E Zeit, v. 3, n. 1, p. 3-16, 1988.

- Verdrängte Vergangenheit. Darstellungstechniken und Deutungen der Fachgeschichte im „Dritten Reich“ in den Personalien der „Publizistik“. In: HOLTZ-BACHA, Christina; KUTSCH, Arnulf; LANGENBUCHER, Wolfgang; SCHÖNBACH, Klaus. 50 Jahre Publizistik. Wiesbaden: VS, p. 73-112, 2006.

. Die Entstehung des Deutschen Zeitungswissenschaftlichen Verbandes. In: Jahrbuch für Kommunikationsgeschichte, v. 12, p. 120-144, 2010.

. Kommunikations- und Medienwissenschaft. In: VON HEHL, Ulrich; JOHN, Uwe; RUDERSDORF, Manfred. Geschichte der Universität Leipzig 1409-2009, v. 4/1. Leipzig: Universitätsverlag, p. 741-759, 2009.

; HEMELS, Joan; SCHMOLKE, Michael. Entgrenzungen. Erinnerungen an Henk Prakke. Assen: Van Gorcum, 2000.

KURTH, Karl; HOLLMANN, Wolfgang. Die Wirkungsgesetze der Presse. Gedanken über den Zusammenhang von Volkstum und Nachrichtengestaltung. Essen: Essener Verlagsanstalt, 1940.

KNIEFACZ, Katharina. Zeitungswissenschaft in Wien 1900-1945. Die Institutionalisierung im Kontext der deutschen Fachentwicklung. Diploma Thesis University of Vienna. Disponível em: <http://othes.univie. ac.at/1046/1/2008-08-08_0000987.pdf> . Acesso em: 6 fev. 2014.

LACASA, Ivan. Zeitungswissenschaft als publizistische Aktion? Karl d'Ester, Emil Dovifat, Erich Everth. In: Medien E Zeit, v. 23, n. 4, p. 4-8, 2008.

LANGENBUCHER, Wolfgang. Paul F. Lazarsfeld. Die Wiener Tradition der empirischen Sozial- und Kommunikationsforschung. München: Ölschläger, 1990.

. (Ed.). Otto Groth. Vermittelte Mitteilung. Ein journalistisches Modell der Massenkommunikation. München: Fischer, 1998.

. Ronneberger war ein Chamäleon“. Wolfgang R. Langenbucher im Gespräch mit Wolfgang Duchkowitsch und Fritz Hausjell zu ,braunen Flecken in der Fachgeschichte, personellen und inhaltlichen Kontinuitäten der nationalsozialistischen Zeitungswissenschaft und terminologischen Eierschalen". In: 
DUCHKOWITSCH, Wolfgang; HAUSJELL, Fritz; SEMRAD, Bernd (Ed.). Die Spirale des Schweigens. Zum Umgang mit der nationalsozialistischen Zeitungswissenschaft. Münster: LIT, 2004. p. 23-37.

LÖBLICH, Maria. Die empirisch-sozialwissenschaftliche Wende in der Publizistik- und Zeitungswissenschaft. Köln: von Halem, 2010.

LÖBLICH, Maria; SCHEU, Andreas. Writing the History of Communication Studies: A Sociology of Science Approach. In: Communication Theory, v. 21, n. 1, p. 1-22, 2011.

MANHEIM, Ernst. Die Träger der öffentlichen Meinung. Studien zur Soziologie der Öffentlichkeit. Leipzig, Brno, Prag: Rohrer, 1933.

MARCUSE, Herbert, [Review of] Ernst Manheim. Die Träger der öffentlichen Meinung. In: Zeitschrift für Sozialforschung, v. 3, n. 1, p. 96-99, 1934.

MATTELART, Armand; MATTELART, Michèle. Histoire des théories de la communication. Paris: La Découverte, 2002.

MEYEN, Michael. Promovieren bei Karl d'Ester. Ein Beitrag zur Frühgeschichte der Zeitungswissenschaft in Deutschland. In: MEYEN, Michael; LÖBLICH Maria (eds.). 80 Jahre Zeitungs- und Kommunikationswissenschaft in München. Bausteine zu einer Institutsgeschichte. Köln: von Halem, p. 28-45, 2004.

(2013). Fachgeschichte als Generationengeschichte. Disponível em: <http://blexkom.halemverlag.de/ fachgeschichte-im-schnelldurchlauf/>. Acesso em: 5 fev. 2014.

MEYEN, Michael; LÖBLICH, Maria: Klassiker der Kommunikationswissenschaft. Fach- und Theoriengeschichte in Deutschland. Konstanz: UVK, 2006.

MÜNZNER, Gerhard. Presse und öffentliche Meinung. Eine sozialwissenschaftliche Studie. Karlsruhe: Braun, 1928.

Jewish Labour Economy In Palestine. The Economic acitivities of the General Federation of Jewish Labour (Histradruth Ha'ovdim). Jerusalem, Tel Aviv: Economic Research Institute of the Jewish Agency for Palestine,1943.

PÖTTKER, Horst. Öffentlichkeit als gesellschaftlicher Auftrag. Klassiker der Sozialwissenschaft über Journalismus und Medien. Konstanz: UVK, ANO.

. Öffentlichkeit als gesellschaftlicher Auftrag. Klassiker der Sozialwissenschaft über Journalismus und Medien. Konstanz: UVK, 2001.

. Konformität - Opportunismus - Opposition. Zur Typologie von Verhaltensweisen im NS-Regime und danach. In: DUCHKOWITSCH, Wolfgang; SEMRAD, Bernd; HAUSJELL, Fritz. Die Spirale des Schweigens. Zum Umgang mit der nationalsozialistischen Zeitungswissenschaft. Münster: LIT, 2004. S. 51-45. 
REIMANN, Horst. Paul Lazarsfeld und die Entstehung der Massenkommunikationsforschung als Verbindung amerikanischer und europäischer Forschungstraditionen. In: LANGENBUCHER, Wolfgang. Paul F. Lazarsfeld. Die Wiener Tradition der empirischen Sozial- und Kommunikationsforschung. München: Ölschläger, 1990. p. 112-130.

ROWLAND, Allison; SIMONSON, Peter. The Founding Mothers of Communication Research: Toward a History of a Gendered Assemblage. In: Critical Studies in Media and Communication, 2013.

SCANNELL, Paddy. Media and Communication. Los Angeles: Sage, 2007.

SCHÄFER, Fabian. Public Opinion, Propaganda and Ideology. Theories on Press and its Social Function in Interwar Japan, 1918-1937. Leiden, Boston: Brill, 2012.

SCHENK, Michael. Medienwirkungsforschung. Tübingen: Mohr Siebeck, 2007.

SCHÜTZ, Walter. Neuanfang mit brauner Lektüre. Studienbedingungen nach 1945. Ein Erfahrungsbericht. In: DUCHKOWITSCH, Wolfgang; SEMRAD, Bernd; HAUSJELL, Fritz. Die Spirale des Schweigens. Zum Umgang mit der nationalsozialistischen eitungswissenschaft. Münster: LIT, 2004. S. 145-123.

SIMPSON, Christopher. Elisabeth Noelle-Neumann's Spiral of Silence and the Historical Context of Communication Theory. Journal of Communication, v. 46, n. 3, p. 149-171, 1997.

SIX, Franz-Alfred (1936). Die Politische Propaganda der NSDAP im Kampf um die Macht. SÖSEMANN, Bernd (Ed.). Emil Dovifat. Studien zu Leben und Werk. In Zusammenarbeit mit Gunda Stöber. Berlin: Walter de Gruyter, 1998.

STÖBER, Rudolf. Karl d'Ester und die Frühgeschichte der deutschen Presse. Das Zeitungswesen in Westfalen von den ersten Anfängen bis zum Jahr 1813. Münster 1907. In: MEYEN, Michael; LÖBLICH, Maria. 80 Jahre Zeitungs- und Kommunikationswissenschaft in München. Bausteine zu einer Institutsgeschichte. Köln: von Halem, 2004a. p. 20-45.

. Emil Dovifat, Karl d'Ester und Walter Hagemann. In: DUCHKOWITSCH, Wolfgang; HAUSJELL, Fritz; SEMRAD, Bernd. Die Spirale des Schweigens. Zum Umgang mit der nationalsozialistischen Zeitungswissenschaft. Münster: LIT, 2004b. p. 123-143.

THUSSU, Daya Kishan. Internationalizing Media Studies. London: Routledge, 2009.

URBAN, Regina; HERPOLSHEIMER, Ralf. Franz Alfred Six (geb. 1909). In: KUTSCH, Arnulf. Zeitungswissenschaftler im Dritten Reich. Sieben biographische Studien. Köln: Hayit, 1984. p. 169-214.

WAGNER, Hendrik. Hans Amandus Münster. In: MEYEN, Michael; WIEDEMANN, Thomas (Ed.). Biografisches Lexikon der Kommunikationswissenschaft. 2013. Disponível em: <http://blexkom.halemverlag.de/ hans-amandus-munster/>. Acesso em: 5 fev. 2014. 
WENDELIN, Manuel. Elisabeth Noelle-Neumann. In: MEYEN, Michael;WIEDEMANN, Thomas (Ed.). Biografisches Lexikon der Kommunikationswissenschaft. 2013. Disponível em: <http://blexkom.halemverlag.de/ elisabeth-noelle-neumann/>. Acesso em: 6 fev. 2014.

WIEDEMANN, Thomas. Walter Hagemann. Aufstieg und Fall eines politisch ambitionierten Journalisten und Publizistikwissenschaftlers. Köln: von Halem, 2012.

WEISCHENBERG, Siegfried. Max Weber und die Entzauberung der Medienwelt. Theorien und Querelen - eine andere Fachgeschichte. Wiesbaden: Springer VS, 2012.

WILLEMS, Emil. Kollektivmeinung und Presse in Zusammenhängen. Ein Beitrag zur speziellen Soziologie. Köln: Pilgram, 1930.

\section{NOTAS}

1 Tradução de Francisco Rüdiger.

2 Veja Averbeck \& Kutsch (2002), reimpresso e recapitulado em Meyen \& Löblich (2006, p. 40-41), e disponível em inglês em Averbeck (2008, p. 4-5).

3 Veja Kutsch (1988), Pötkker (2001, p. 313), Meyen; Löblich (2006, p. 145) e Weischenberg (2012, p. 78).

4 Veja as resenhas de seu livro sobre a opinião pública feitas por Wilhelm Kapp, professor de jornalismo, e pelo filósofo Herbert Marcuse, em 1934.

5 Sobre a história do Instituto de Leipzig durante a República de Weimar, o estado nazista e a República Democrática Alemã até 1989, veja Kutsch (2009).

6 A respeito destes tipos de pesquisa, conferir Meyen; Löblich (2006, p. 15); Löblich; Scheu (2011), Meyen (2013).

7 Confira a respeito em Duchkowitsch; Hausjell; Semrad (2004).

Recebido em: 15 maio 2014

Aceito em: 15 jun. 2014

\section{Endereço da autora:}

Stefanie Averbeck-Lietz <averbeck.lietz@uni-bremen.de>

Linzer Strasse 4

1400, Bremen, Alemanha 